\title{
Carrier Lifetime and Mobility Characterization using the DTU 3D CZT Drift Strip Detector
}

Howalt Owe, S.; Kuvvetli, I.; Budtz-Jorgensen, C.

Published in:

IEEE Transactions on Nuclear Science

Link to article, DOI:

10.1109/TNS.2021.3068001

Publication date:

2021

Document Version

Peer reviewed version

Link back to DTU Orbit

Citation (APA):

Howalt Owe, S., Kuvvetli, I., \& Budtz-Jorgensen, C. (2021). Carrier Lifetime and Mobility Characterization using the DTU 3D CZT Drift Strip Detector. IEEE Transactions on Nuclear Science, 68(9), 2440-2446.

https://doi.org/10.1109/TNS.2021.3068001

\section{General rights}

Copyright and moral rights for the publications made accessible in the public portal are retained by the authors and/or other copyright owners and it is a condition of accessing publications that users recognise and abide by the legal requirements associated with these rights.

- Users may download and print one copy of any publication from the public portal for the purpose of private study or research.

- You may not further distribute the material or use it for any profit-making activity or commercial gain

- You may freely distribute the URL identifying the publication in the public portal

If you believe that this document breaches copyright please contact us providing details, and we will remove access to the work immediately and investigate your claim. 


\title{
Carrier Lifetime and Mobility Characterization using the DTU 3D CZT Drift Strip Detector
}

\author{
S. Howalt Owe, I. Kuvvetli, and C. Budtz-Jørgensen
}

\begin{abstract}
At DTU Space a 3D CdZnTe (CZT) drift strip detector prototype of size $20 \mathrm{~mm} \times 4.7 \mathrm{~mm} \times 20 \mathrm{~mm}$ has been developed. It has demonstrated excellent submillimeter position resolution $(<0.5 \mathrm{~mm})$, and energy resolution $(<1.6 \%)$ at $661.6 \mathrm{keV}$ using pulse shape signal processing. Signal formation on each of the 26 electrode readouts uses bi-polar Charge Sensitive Pre-amplifiers. The output is sampled using high speed digitizers; providing us with the full pulse shapes generated by each interaction in the detector. In order to optimize and understand the detector performance, a model of the 3D CZT drift strip detector has been developed using COMSOL Multiphysics ${ }^{\circledR}$ and Python. It simulates the 26 pulse shapes generated by an interaction, and provides an output similar to that of the real detector setup. In order to create a trustworthy model, the material properties of the detector must be well understood. The generated pulse shapes are greatly affected by the electron mobility $\left(\mu_{e}\right)$ and lifetime $\left(\tau_{e}\right)$ of the detector material. Therefore, 3D maps of $\mu_{e}$ and $\tau_{e}$ have been calculated as look-up tables for the model, utilizing the high resolution 3D interaction position and energy information provided by the 3D CZT drift strip detector. In conclusion, the model performance is compared to real event data. We show that the model performance is greatly improved using the newly calculated 3D maps compared to the uniform material properties provided by the crystal manufacturer.
\end{abstract}

Index Terms-3D CZT detectors, CZT Drift Strip Detectors, Digitized pulse shape analysis, Carrier lifetime and mobility

\section{INTRODUCTION}

Pulse shape formation in a semiconductor detector is greatly affected by the electrode geometry (weighting potential effect), and the charge carrier mobility $(\mu)$ and lifetime $(\tau)$ of the detector material. To build a reliable model for simulation of the pulse shape formation within a semiconductor detector, the detector material properties must be well understood. A method for determining the electron mobility $\left(\mu_{e}\right)$ and lifetime $\left(\tau_{e}\right)$ has previously been described [1] [2], estimating uniform material properties of the detector. In this paper, we investigate how to use these methods to create a 3D map of $\mu_{e}$ and $\tau_{e}$ within the 3D CdZnTe (CZT) drift strip detector utilizing its 3D position and spectroscopic capabilities. The material nonuniformities will thereby be taken into account. The 3D maps are then implemented as look-up tables in the 3D CZT detector model, and its impact on the model performance investigated.

High resolution 3D CZT drift strip detectors are showing promising results within both medical and astrophysical applications [3] [4] [5]. The CZT drift strip detector principle,

S. Howalt Owe is a PhD student at the Technical University of Denmark, Kgs. Lyngby, DK-2800, Denmark (e-mail: shoowe@space.dtu.dk).

I. Kuvvetli is a senior scientist at the Technical University of Denmark, Kgs. Lyngby, DK-2800, Denmark (e-mail: irfan@space.dtu.dk).

C. Budtz-Jørgensen is a senior scientist emeritus at the Technical University of Denmark, Kgs. Lyngby, DK-2800, Denmark (e-mail: carl@space.dtu.dk).
[6] [7], screens the anodes from the poor hole movement in CZT, such that the detector performance mainly depend on the electron charge transport characteristics. The high-resolution, 3D position sensitive, CZT detector prototype developed at DTU Space, is of size $20 \mathrm{~mm} \times 4.7 \mathrm{~mm} \times 20 \mathrm{~mm}$. The CZT crystal was manufactured by REDLEN, and details on the detector prototype fabrication can be found in [8]. On one plane of the detector 10 cathodes are deposited, and on the other side 12 anodes and 37 drift strips are deposited perpendicular to the cathodes. The drift strips are sectioned into four readouts, resulting in four drift strip sections. At standard operation the anodes are grounded, the cathodes are biased at $-350 \mathrm{~V}$, and the drift strips are biased at $-120 \mathrm{~V}$ (central drift strips are held at a bias of $2 / 3$ of $-120 \mathrm{~V}$ and the two adjacent drift strips are held at $1 / 3$ of $-120 \mathrm{~V}$, such that electrons are drifted towards collection at the anode). See Fig. 1 for an electrical diagram of the 3D CZT detector.

The readout technique and the developed interaction position reconstruction algorithms have resulted in excellent 3D position, and energy resolution for high energy photon interactions [9]. The system has currently been tested at $661.6 \mathrm{keV}$ using a ${ }^{137} \mathrm{Cs}$ source. It demonstrated a position resolution of $0.5 \mathrm{~mm} \times 0.4 \mathrm{~mm} \times 0.5 \mathrm{~mm}$ in the $\mathrm{x}-, \mathrm{y}-$, and z-direction respectively, and an energy resolution of $<1.6 \%$ [5]; the current setup suffers from high electronic noise (5$7 \mathrm{keV}$ FWHM) and is therefore the limiting factor to this resolution. The high-speed digitizers enable sampling of the full pulse shape formation of the signal; see Fig. 2 for an example. The pulse shapes of the given interaction contain substantial information about the interaction, for example, 3D interaction position, energy deposition, electron drift time, and interaction type (e.g. photoelectric absorption event, or a Compton interaction event). 3D position information in the detector, in combination with the knowledge of interaction types, makes this detector an excellent candidate for future high energy Compton camera applications [5]. Understanding how the signals are generated within the detector is a key factor for further development and improvements.

To optimize and understand the detector performance, a model of the 3D CZT drift strip detector has been developed using COMSOL Multiphysics ${ }^{\circledR}$ and Python. The 3D CZT detector electrode geometry was implemented in COMSOL in order to calculate the electrostatic conditions of the detector, as input for the charge movement and transport Python script. The detector model can be configured by a set of input parameters, such as sample time, sample length, and start time and initial 3D position of the photon interaction. The detector model calculates the induced signals on all 26 electrodes, and 


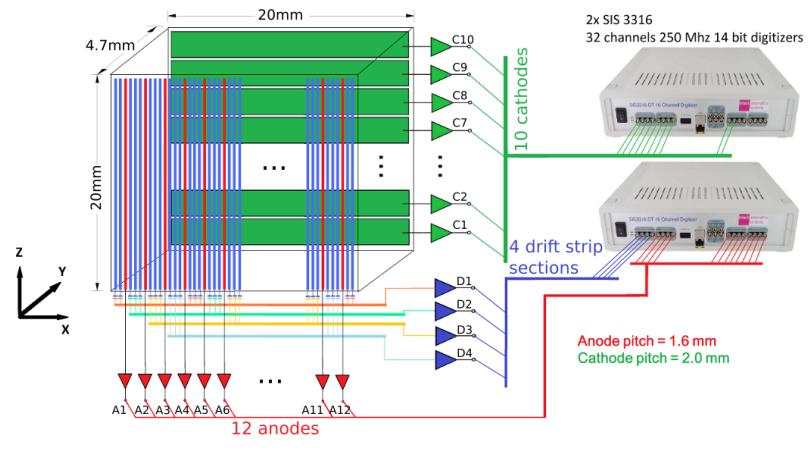

Fig. 1. Electrical diagram of the 3D CZT drift strip detector.
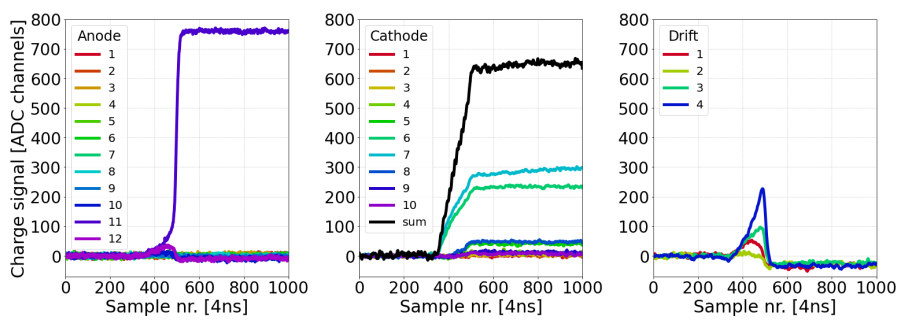

Fig. 2. Example of 26 pulse shapes measured by the 3D CZT drift strip detector system. Left: 12 anode signals. Center: 10 cathode signals and the sum of the cathode signals. Right: Four drift strip signals.

returns the 26 generated pulse shapes for the given interaction position. For comparison purpose, the output is provided in a data format similar to the one for the real detector setup using digitizers.

The current model uses uniform material properties for the entire detector volume. Since the pulse shape formation is greatly affected by $\mu_{e}$ and $\tau_{e}$ of the detector material, it is imperative that the values used by the model resembles the ones from the true detector. If not, the simulated pulse shapes and the true pulse shapes are incomparable.

\section{MATERIALS AND METHOD}

To investigate the $\mu_{e}$ and $\tau_{e}$ parameters throughout the 3D CZT detector volume, three estimations are produced. First, we estimate the product $\mu_{e} \tau_{e}$, next we estimate the electron lifetime $\tau_{e}$, and finally, we use the two estimations to isolate the electron mobility $\mu_{e}$. For the analysis we utilize the 3D CZT detector data. All single event interactions are extracted, and for each of these events the following information is determined using pulse shape analysis:

1) $3 \mathrm{D}$ position of the event in the $3 \mathrm{D}$ CZT detector.

2) Deposited energy by the photon in the $3 \mathrm{D}$ CZT detector.

3) Drift time of the generated electron charge cloud.

The 3D position information is used to divide the detector volume into a virtual grid of data volumes (i.e. voxels), to each of which a pair of $\mu_{e}$ and $\tau_{e}$ values are associated, utilizing the events occurring within the voxel. In order to do this, the deposited energy and electron drift time is used, extracted from measurements taken at different voltage bias settings, resulting in a change in the electric field strength in between each measurement.

\section{A. Experimental setup}

To determine $\mu_{e}$ and $\tau_{e}$ of the detector material, sets of data collected at varying voltage bias $(V)$ between the anodes and cathodes are used. The anodes are grounded and the drift strips and cathodes are biased in a total of six configurations varying the cathode bias from $-150 \mathrm{~V}$ to $-400 \mathrm{~V}$. For each cathode bias the drift bias was chosen to be a 3rd of the cathode bias, with a maximum bias of $-120 \mathrm{~V}$, due to electronic noise. See Table I for an overview of the measurement voltage settings. A ${ }^{137} \mathrm{Cs}(661.6 \mathrm{keV})$ source was placed, illuminating the entire XZ-plane (anode plane) of the detector, at a distance of 20 $\mathrm{cm}$. For each bias setting a set of 20 hour measurements were taken, resulting in a total of 120 hours of data collection.

TABLE I

CATHODE AND DRIFT VOLTAGE BIAS SETTINGS.

\begin{tabular}{ccc}
\hline Measurement & Cathode bias [V] & Drift bias [V] \\
\hline 1 & -150 & -50 \\
2 & -200 & -66 \\
3 & -250 & -83 \\
4 & -300 & -100 \\
5 & -350 & -120 \\
6 & -400 & -120 \\
\hline
\end{tabular}

\section{B. Estimating the electric field strength}

The change in voltage bias between each measurement is done in order to vary the electric field strength $(E)$ inside the detector. This affects the movement of the electron charge cloud, which will drift quicker towards collection and experience less electron charge trapping as the electric field strength increase. We know the bias voltage values exactly, but how do we then make a proper estimation of the electric field strength within the detector? When it comes to the electron drift path, the 3D CZT drift strip detector can be assumed as a planar detector for most of the detector volume. See Fig. 3, which illustrate the electric field path within one drift cell of the 3D CZT detector simulated using COMSOL. A uniform electric field path is seen throughout most of the detector medium, only close to the anode side $(y<1 \mathrm{~mm})$ we see this assumption will no longer hold. For this analysis we have chosen to model and estimate the electric field strength and shape by simulation using COMSOL.

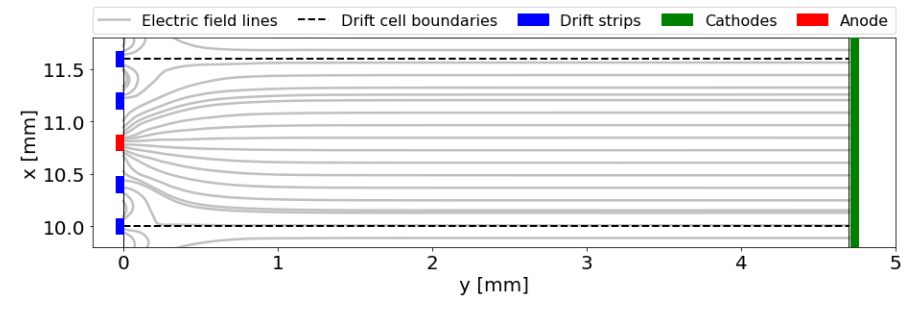

Fig. 3. COMSOL simulation and illustration of the electric field lines zoomed in on a single drift cell in the 3D CZT drift strip detector at a cathode bias voltage of $-350 \mathrm{~V}$ and a drift bias voltage of $-120 \mathrm{~V}$. 


\section{Estimation of the $\mu_{e} \tau_{e}$-product}

The $\mu_{e} \tau_{e}$-product is estimated utilizing the electric field strengths, electron drift distance, and photopeak position using the relation [1],

$$
N=N_{0} \exp \left(-\frac{y_{d}}{\mu_{e} \tau_{e} E}\right)
$$

where $N$ is the number of electrons collected after a drift distance of $y_{d}, E$ is the electric field strength in the detector, and $N_{0}$ is the original number of electron-hole pairs. Consider the two cases where measurements are taken at two different bias voltages between anode and cathode $\left(V_{1}\right.$ and $\left.V_{2}\right)$. This will result in two different electric field strengths $\left(E_{1}\right.$ and $\left.E_{2}\right)$, and two different measured photopeak amplitudes $\left(N_{1}\right.$ and $N_{2}$ ) as the photopeak amplitudes are proportional to the number of electrons collected at the anode surface. Using this, two expressions of (1) can be used to solve and get rid of $N_{0}$ such that [1]

$$
\ln \left(\frac{N_{1}}{N_{2}}\right)=\frac{y_{d}}{\mu_{e} \tau_{e}}\left(\frac{1}{E_{2}}-\frac{1}{E_{1}}\right)
$$

where $y_{d}$ is the electron drift length for data with photopeak amplitudes $N_{1}$ and $N_{2}$ [1]. Both the measurement of $N_{1}$ and $N_{2}$ have the same non-linear weighting potential effect on the signals as a function of $y_{d}$; as previously described [9]. The ratio of the two signals given in (2) will cancel out this effect.

The six measurements (Table I) are then used to calculate five points. $N_{1}$ is described by the data measured at biases from measurement number 1 to 5 , and $N_{2}$ is described by the data measured at the highest bias (measurement 6). Plotting $\ln \left(N_{1} / N_{2}\right)$ as a function of $\left(1 / E_{2}-1 / E_{1}\right)$ and fitting a linear regression model, results in the slope,

$$
\alpha=\frac{y_{d}}{\mu_{e} \tau_{e}}
$$

and knowing the electron drift length $y_{d}$, we can estimate the $\mu_{e} \tau_{e}$-product. For an estimation of the error in the calculated $\mu_{e} \tau_{e}$-product error propagation is applied to (2). The electric field strengths are assumed to be known accurately, which results in the uncertainty in $N_{1}, N_{2}$, and $y_{d}$ contributing to the error.

\section{Estimation of the electron lifetime $\tau_{e}$}

$\tau_{e}$ is estimated utilizing electron drift times together with photopeak positions measured at different voltage bias between anode and cathode. Given the decay of the freecarrier concentration as a function of time, and assuming that the collected charge is proportional to the amplitude of the photopeak position, we have for two measurements at different bias voltage settings [2]

$$
\begin{aligned}
& N_{1}=N_{0} \exp \left(-\frac{t_{d 1}}{\tau_{e}}\right) \\
& N_{2}=N_{0} \exp \left(-\frac{t_{d 2}}{\tau_{e}}\right)
\end{aligned}
$$

where $t_{d 1}$ is the electron drift time from the initial interaction position at the first bias setting, and $t_{d 2}$ is the electron drift time from the initial interaction position at the second bias setting. Solving using (4) and (5), we can again cancel out the original number of electron-hole pairs $N_{0}$,

$$
\ln \left(\frac{N_{1}}{N_{2}}\right)=\frac{1}{\tau_{e}}\left(t_{d 2}-t_{d 1}\right) .
$$

The six measurements (Table I) are then used to calculate five points. $N_{1}$ and $t_{d 1}$ is described by the data measured at biases from measurement number 1 to 5 , and $N_{2}$ and $t_{d 2}$ is described by the data measured at the highest bias (measurement 6). Plotting $\ln \left(N_{1} / N_{2}\right)$ as a function of $\left(t_{d 2}-t_{d 1}\right)$ and fitting a linear regression model, results in the slope,

$$
\alpha=\frac{1}{\tau_{e}}
$$

allowing us to estimate the electron lifetime $\tau_{e}$. For an estimation of the error in the calculated $\tau_{e}$, error propagation is applied to (6). The error calculation will be affected by the uncertainties of the estimated energies and electron drift times, $N_{1}, N_{2}, t_{d 1}$, and $t_{d 2}$. The uncertainty of the estimated energies are mostly affected by the energy resolution of the detector, where the uncertainties of the electron drift times are strongly affected by the voxel size. Especially close to drift cell boundaries, electron drift times will vary more, compared to directly above the anode.

\section{E. Estimation of the electron mobility $\mu_{e}$}

$\mu_{e}$ is lastly estimated from the determined values of $\mu_{e} \tau_{e}$ and $\tau_{e}$,

$$
\mu_{e}=\frac{\mu_{e} \tau_{e}}{\tau_{e}} .
$$

The error estimation is again done using error propagation applied to (8) with the estimated errors in $\mu_{e} \tau_{e}$ and $\tau_{e}$ contributing.

\section{DATA PREPARATION}

For each of the six bias setting measurements, the 26 pulse shapes for each photon interaction were processed, with only single trigger event data considered. This resulted in a dataset containing the following information for each photon interaction: 3D position, $26 \mathrm{ADC}$ pulse heights, and electron drift time.

\section{A. Position determination}

The position determination algorithms will be briefly described in this section. Further information on the positioning algorithms can be found in [5] and [9].

1) x-position: The x-position in the 3D CZT detector is along the direction of the anodes; see Fig. 1. For the $\mathrm{x}$ position determination we utilize the peak value of the two adjacent inverted drift strip current signals to the triggered anode, denoted $A_{D L}$ and $A_{D R}$. Coupled with this information, the triggered anode number $x_{\text {trig }}$ is used to determine the $\mathrm{x}$ position,

$$
x=0.4+\left[x_{t r i g}+F \cdot \frac{A_{D R}-A_{D L}}{A_{D R}+A_{D L}}\right] \cdot P_{a n}-\frac{P_{a n}}{2},
$$


where the constant 0.4 is the initial distance from the edge to the first electrode, $F$ is an adjustable factor for calibration of the x-scale, currently set to 0.69 , and $P_{a n}$ is the anode pitch of $1.6 \mathrm{~mm}$.

2) y-position: The y-position of the detector describes the depth of the interaction between the anode side, and cathode side of the detector, see Fig. 1. When determining the yposition, the cathode pulse heights, $C_{n}$, are summed and divided by the pulse height of the triggered anode $A_{m}$. This returns the depth parameter of the detector, which is then calculated in units of detector thickness, $D$,

$$
y=\frac{\sum_{n=1}^{10} C_{n}}{A_{m}} \cdot D .
$$

3) z-position: The z-position is determined utilizing the 10 cathode amplitudes in a center of gravity of cathode strip calculation. Utilizing the cathode pulse heights, $C_{n}$, and the cathode pitch, $P_{c a}$, of $2 \mathrm{~mm}$, the z-position is given by,

$$
z=\frac{\sum_{n=1}^{10} n \cdot C_{n}}{\sum_{n=1}^{10} C_{n}} \cdot P_{c a} .
$$

\section{B. Pulse height determination}

All 26 pulse shapes are adjusted to have the same baseline (see Fig. 2). The cathode and anode pulse heights are simply determined by subtracting the average value of the first 150 samples from the average value of the last 150 samples. The drift strip signals are bi-polar, and therefore the pulse heights are not extracted. However, the peak position of each derivative of the pulse shape (current profile) is extracted, and used when determining the $\mathrm{x}$-position.

\section{Drift time determination}

The electron drift time of each event is determined by the sample time of the beginning, $t_{0}$, and the end, $t_{1}$, of the event. To determine these, the sum of the cathode signal pulse shapes is utilized. The cathode sum is obtained by summing the 10 cathode pulse shapes after readout. Fig. 4 shows an example of the summed cathode signal of an arbitrary event. The cathodes will see the electron cloud movement at the instant the charge cloud starts to drift. This results in an increase in the induced signal. The pulse shape will continue increasing until the electron charge cloud is collected, where the pulse shape once again will flatten. The beginning and end of the event can then be determined by applying a linear fit to the slope of the signal. The point where the linear fit intersects with the baseline, is chosen as the beginning of the event, $t_{0}$. The point where the linear fit intersects with the point where the cathode signal sum flattens is chosen as the end of the event $t_{1}$. The two time values, $t_{0}$ and $t_{1}$, can then be used to calculate the drift time, $t_{d}$. The unit of these values are the sample time $4 n s$. For events close to the anode side, the created cathode pulses will be characterized by small pulse heights. This can result in the determined drift time being distorted. Therefore, this method is not covering events close to the anode side.

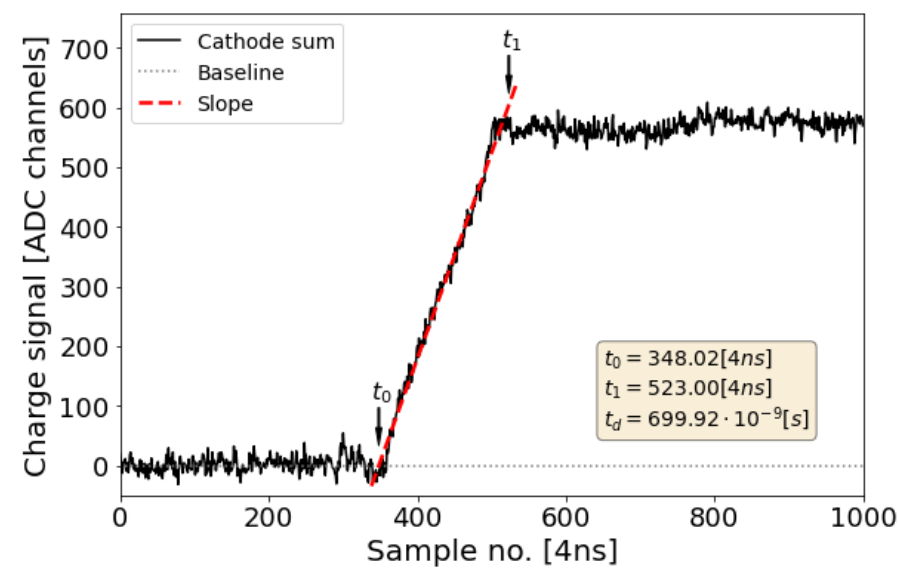

Fig. 4. Illustration of the procedure of utilizing the sum of cathode pulse shapes to determine the start time, $t_{0}$, and end time, $t_{1}$, of the event. The drift time in seconds can then be calculated by $t_{d}=4 \cdot\left(t_{1}-t_{0}\right) \cdot 10^{-9}$.

\section{The dataset}

For each measurement of all voltage bias settings, the pulse heights, 3D positions, and drift times, are extracted using the methods described in the previous sections. This resulted in six data sets; one for each voltage bias setting. Fig. 5 shows 2D-histograms of the XZ-plane and the XY-plane of the 3D position information for the measurement at cathode voltage bias $-350 \mathrm{~V}$ and drift voltage bias of $-120 \mathrm{~V}$. The XZ-plane shows no events for $z<2 \mathrm{~mm}$ and $z>18 \mathrm{~mm}$, due to more severe edge effect on the signals. Furthermore, we do see areas where there are fewer or no events around anodes 5 (A5), 6 (A6), 11 (A11), and 12 (A12) for $z<5.0 \mathrm{~mm}$, as encountered earlier [5]. Investigating the XY-plane we see the same artefacts as earlier encountered [5], [9], with anode 1 (A1) and 12 (A12) showing clear edge effects and the $\mathrm{x}$ position algorithm not covering events with $y<1 \mathrm{~mm}$.

Investigating the 2D-histograms of the XY- and XZ-plane, a subset region was chosen for this analysis. The subset considered in this analysis is made only of anodes 2-11 with the $\mathrm{y}$-position limits $2.0 \mathrm{~mm} \leq y \leq 4.0 \mathrm{~mm}$, and the $\mathrm{z}$ position limits $13.0 \mathrm{~mm} \leq z \leq 15.0 \mathrm{~mm}$. Furthermore, along the $\mathrm{x}$-direction, only data within $0.2 \mathrm{~mm}$ from each drift cell boundary is considered. The white highlighted areas in Fig. 5 indicate the data subset used for this analysis. The subvolume is then divided into virtual volumes, each of size $(d x, d y$, $d z)=(0.4 \mathrm{~mm}, 0.2 \mathrm{~mm}, 1.0 \mathrm{~mm})$. The size is mainly limited by statistics. If we wish to make the volumes even smaller, more data must be collected. However, for the aim of the current analysis this volume size is sufficient. We have chosen to minimize $d y$ since we wish to determine the drift time as precisely as possible. Table II shows an overview of data division boundaries. The analysis can be expanded to include the entire detector volume at a later time.

\section{DATA ANALYSIS}

\section{A. Determination of pulse heights and drift time}

Each data set (Table I), all followed the same data analysis procedure, described in Section III. Using the 3D position 

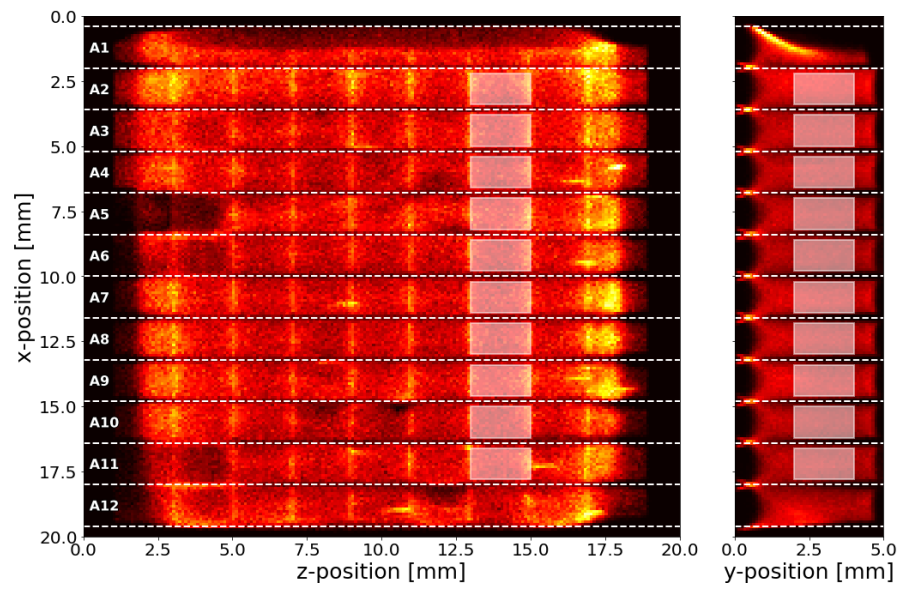

Fig. 5. 2D-histograms displaying the calculated $3 \mathrm{D}$ positions for the measurement taken with a cathode bias of $-350 \mathrm{~V}$ and a drift bias of $-120 \mathrm{~V}$. White dashed lines indicates drift cell boundaries. Anode numbers are defined within each drift cell. The white highlighted areas indicate the chosen data subset of the detector volume.

TABLE II

OVERVIEW OF THE 3D DATA DIVISION BOUNDARIES

\begin{tabular}{|c|c|c|c|c|}
\hline Direction & $\begin{array}{l}\text { Number } \\
\text { of slices }\end{array}$ & $\begin{array}{l}\text { Slice thickness } \\
{[\mathrm{mm}]}\end{array}$ & $\begin{array}{l}\text { Minimum } \\
\text { value }[\mathrm{mm}]\end{array}$ & $\begin{array}{l}\text { Maximum } \\
\text { value }[\mathrm{mm}]\end{array}$ \\
\hline $\mathrm{x}($ anode 2$)$ & & & 2.2 & 3.4 \\
\hline$x$ (anode 3 ) & 1 & & 3.8 & 5.0 \\
\hline$x$ (anode 4) & & & 5.4 & 6.6 \\
\hline $\mathrm{x}$ (anode 5) & $r$ & & 7.0 & 8.2 \\
\hline$x$ (anode 6) & & & 8.6 & 9.8 \\
\hline x (anode 7) & 3 & 0.4 & 10.2 & 11.4 \\
\hline $\mathrm{x}($ anode 8$)$ & & & 11.8 & 13.0 \\
\hline $\mathrm{x}($ anode 9$)$ & & & 13.4 & 14.6 \\
\hline $\mathrm{x}$ (anode 10$)$ & 1 & & 15.0 & 16.2 \\
\hline $\mathrm{x}$ (anode 11) & & & 16.6 & 17.8 \\
\hline $\mathrm{y}$ & 10 & 0.2 & 2.0 & 4.0 \\
\hline $\mathrm{Z}$ & 2 & 1.0 & 13.0 & 15.0 \\
\hline
\end{tabular}

information, each data set was divided into the virtual volumes described by Table II. To calculate $\mu_{e}$ and $\tau_{e}$ following the methods described in Section II, it is essential to determine a pulse height, drift time, and electron drift distance representing each volume element (or voxel). Therefore, for each voxel, a histogram of the photopeak and electron drift time distribution was plotted, and fitted with a Gaussian distribution. The peak position of the fit was chosen to represent the pulseheight position and electron drift time for the volume. Fig. 6 shows an example of this fitting procedure. Here we see data collected by anode number 2 , for the cathode voltage bias of $-350 \mathrm{~V}$, for $\mathrm{y}$-slice number 4 , $\mathrm{z}$-slice number 1 , and all three $\mathrm{x}$-slices.

\section{B. Estimation of the electron drift distance}

The electron drift distance, $y_{d}$, is used to determine the $\mu_{e} \tau_{e}$-product as stated in (2). For a planar detector, the electron drift distance can be determined as the interaction depth (yposition) in the detector, since the drift path is perpendicular to the anode. However, the geometry of the 3D CZT drift strip detector results in the electron drift path bending towards the anodes close to collection; as illustrated in Fig. 3. Therefore, the electron drift distance is only comparable to the y-position for interactions occurring directly above the anode. An overall
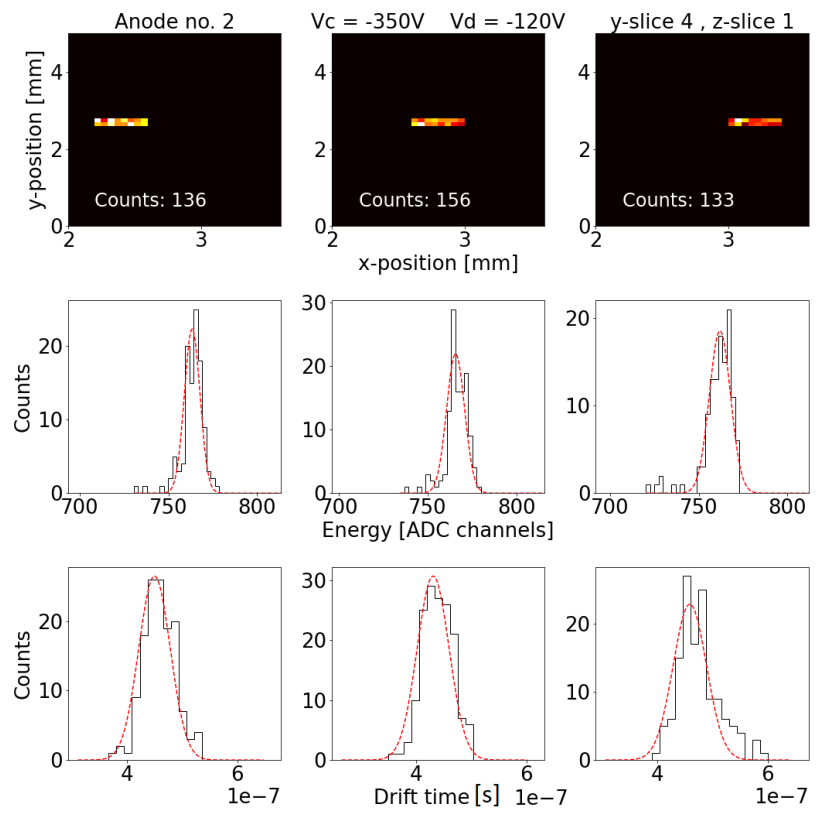

Fig. 6. 2D-position histograms, and Gaussian fit of photopeaks and drift times for three voxels in drift cell number 2, for the measurement settings: Cathode bias $-350 \mathrm{~V}$ and drift bias $-120 \mathrm{~V}$.

estimation of the drift distance for each voxel must therefore be determined, since the volumes closer to the drift cell boundary will have a longer drift path. This was done by investigating data from anode number 8 at a cathode bias of $-350 \mathrm{~V}$ and drift bias of $-120 \mathrm{~V}$. Data along the entire zdirection $(0 \mathrm{~mm}<z<20 \mathrm{~mm})$ and a $0.2 \mathrm{~mm}$ slice along the $\mathrm{y}$-direction $(3.8 \mathrm{~mm}<y<4.0 \mathrm{~mm})$ was used. Along the $\mathrm{x}$ direction $(11.8 \mathrm{~mm}<x<13.0 \mathrm{~mm})$ the data was divided into 11 voxels. For each voxel, the electron drift time was determined. This resulted in 11 values for the electron drift time, where the central value is that of the volume directly above the anode. The drift distance of this volume is assumed to be the central y-position of $3.9 \mathrm{~mm}$. Knowing both drift distance and time, using the central voxel, the electron drift velocity was determined to be

$$
v_{e}=\frac{y_{d}}{t_{d}}=5.94 \cdot 10^{6} \mathrm{~mm} / \mathrm{s} \text {. }
$$

In conjunction with the electron drift velocity, the drift distance of the remaining 10 voxels was estimated using the electron drift time information. In Fig. 7, the resulting difference in drift distance, compared to the central data slice, is shown along with the $\mathrm{x}$-slice boundaries of the material analysis; also defined in Table II. As expected, the interactions that occur closer to the drift cell boundary, will experience a longer drift distance. Values approaching the drift cell boundaries $(0.6 \mathrm{~mm}$ from the drift cell center along the x-direction), will drift a distance of around $0.4 \mathrm{~mm}$ longer than that of the interactions directly above the anode. The central voxel do not have a big variability in electron drift distance. However, events within the boundary volumes vary close to $0.4 \mathrm{~mm}$ in electron drift distance. Since we use the drift distance to determine the $\mu_{e} \tau_{e}$ product in (2), this does impose an uncertainty introduced from the drift distance, especially for the boundary volumes. 
The drift distance representing the boundary volumes was then added a distance of $0.15 \mathrm{~mm}$ (mean difference) compared to the central volume.

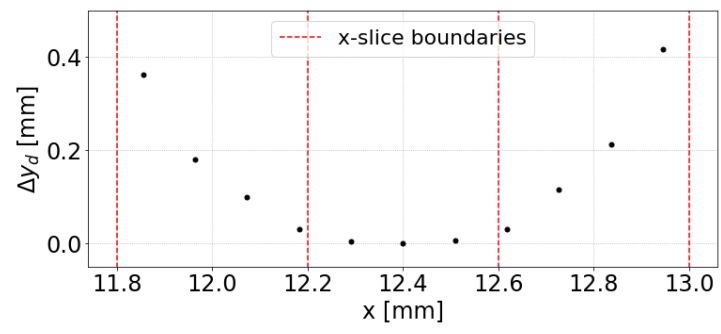

Fig. 7. Difference in drift distances between the an event directly above the anode $(x=12.4 \mathrm{~mm})$ and events closer to the drift cell boundaries. Red dashed lines: Boundaries for the voxels used for the analysis.

\section{RESULTS}

A total of three 3D maps was generated for the chosen detector volume. A map for the $\mu_{e} \tau_{e}$-product, $\tau_{e}$, and $\mu_{e}$. The calculated maps were compared to the original values provided by the manufacturer of the detector crystal given in Table III. In the following sections, the calculated 3D maps will be presented together with a comparison to the manufacturer values.

TABLE III

MATERIAL PROPERTIES OF THE DETECTOR CZT CRYSTAL.

\begin{tabular}{l|lll}
\multicolumn{5}{c}{ MANUfaCtURER } \\
\hline \multicolumn{5}{c}{$\mu \tau\left[\mathrm{cm}^{2} / \mathrm{V}\right]$} & $\tau[\mathrm{s}]$ & $\mu\left[\mathrm{cm}^{2} / \mathrm{Vs}\right]$ \\
\hline Electron & $1.5 \mathrm{e}-2$ & $3.0 \mathrm{e}-5$ & 500 \\
Hole & $0.0006 \mathrm{e}-2$ & $3.0 \mathrm{e}-7$ & 20 \\
\hline \multicolumn{5}{c}{ 3D MAP AVERAGE } \\
\hline \multicolumn{5}{c}{$\mu \tau\left[\mathrm{cm}^{2} / \mathrm{V}\right]$} & $\tau[\mathrm{s}]$ & $\mu\left[\mathrm{cm}^{2} / \mathrm{Vs}\right]$ \\
\hline Electron & $2.9 \mathrm{e}-2 \pm 0.3 \mathrm{e}-2$ & $3.2 \mathrm{e}-5 \pm 1.6 \mathrm{e}-6$ & $909.7 \pm 91$ \\
Hole & - & - & - \\
\hline
\end{tabular}

\section{A. Estimation of the $\mu_{e} \tau_{e}$-product}

The $\mu_{e} \tau_{e}$-map was calculated using the method described in Section II-C for each of the voxels in our 3D map grid. In Fig. 8 the resulting calculated $\mu_{e} \tau_{e}$-map of the chosen detector subvolume is shown. The 3D-map is illustrated in $2 \mathrm{D}$ by showing each of the two slices along the z-direction alone. The upper map is the first z-slice and the lower the second. Using error propagation the mean estimated error of the calculated $\mu_{e} \tau_{e}$-map was determined to be $\sim 10 \%$. We do see a variation in the $\mu_{e} \tau_{e}$-product throughout the detector material, with almost a factor of 3, especially along the $\mathrm{x}$ direction. Thus, we do see variation significantly larger than the estimated error. The variation is of course not provided by the manufacturer, since the manufacturer values assume uniform material properties. Moreover, the presented results are for large parts of the detector significantly larger than the nominal values given in Table III.
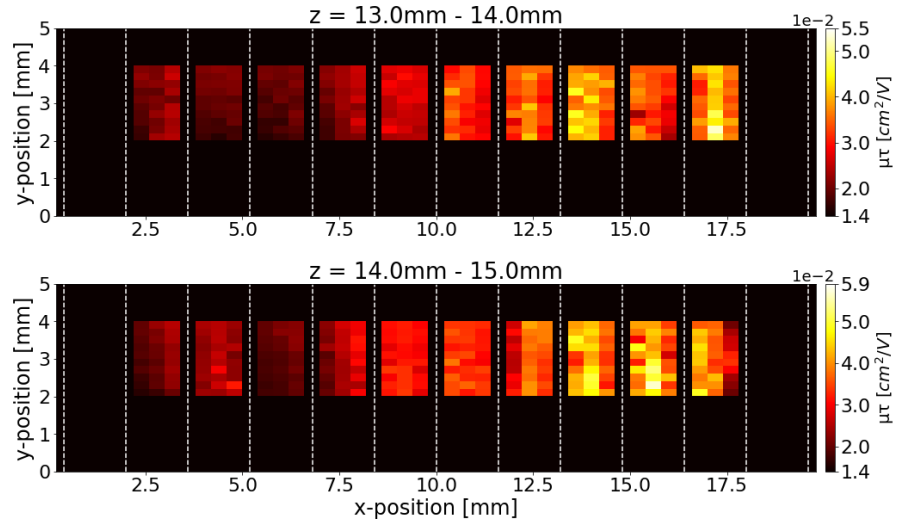

Fig. 8. $\mu_{e} \tau_{e}$-map of the chosen detector subvolume. The mean estimated error of the calculated $\mu_{e} \tau_{e}$-values is $\sim 10 \%$. White dashed lines: Drift cell boundaries.

\section{B. Estimation of the electron lifetime $\tau_{e}$}

The $\tau_{e}$-map was calculated using the method described in Section II-D, also done for each of the voxels in our 3D map grid. In Fig. 9 the calculated $\tau_{e}$-map of the chosen detector subvolume is shown. We identify similar tendencies as for what we observed in the $\mu_{e} \tau_{e}$-map. Using error propagation, the mean estimated error of the calculated $\tau_{e}$-map was determined to be $\sim 5 \%$. Compared to the manufacturer value given in Table III, we see that we are in the same order of magnitude, however with a non-uniformity in the detector material. We note that the variation in $\tau_{e}$ is similar to that of $\mu_{e} \tau_{e}$, and must therefore also be responsible for the variation in $\mu_{e} \tau_{e}$.

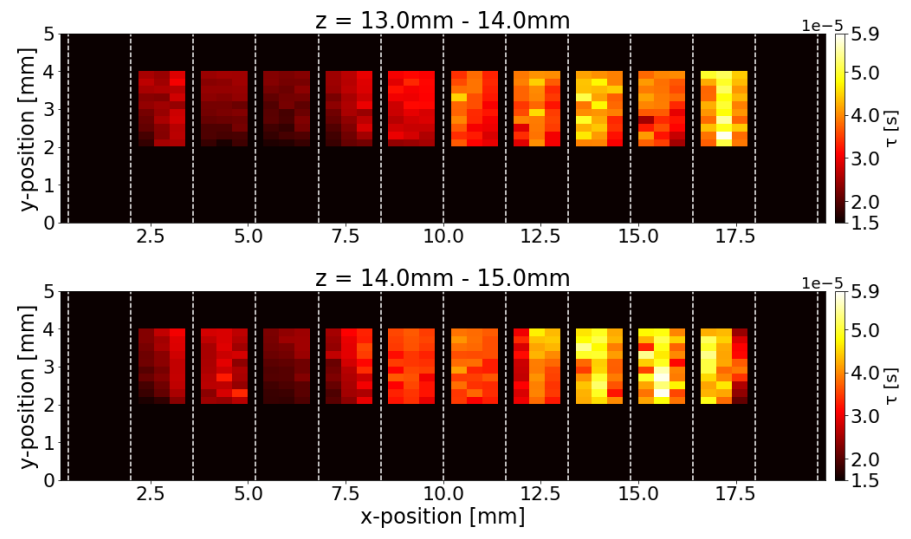

Fig. 9. $\tau_{e}$-map of the chosen detector subvolume. The mean estimated error of the calculated $\tau_{e}$-values is $\sim 5 \%$. White dashed lines: Drift cell boundaries.

\section{Estimation of the electron mobility $\mu_{e}$}

$\mu_{e}$ is estimated using the calculated values for $\mu_{e} \tau_{e}$ and $\tau_{e}$, as described in Section II-E. In Fig. 10 the calculated $\mu_{e^{-}}$ map of the chosen detector subvolume is shown. Using error propagation the mean estimated error of the calculated $\mu_{e}$-map was determined to be $\sim 10 \%$. This error is large compared to the variances we see in the map. Therefore, we cannot draw any conclusions on the material variability, however we can state that the average estimated electron mobility is almost a factor of two larger than the nominal value provided by 
the manufacturer; see Table III. This method estimates the electrons to have a much better mobility than the uniform manufacturer estimation provides.
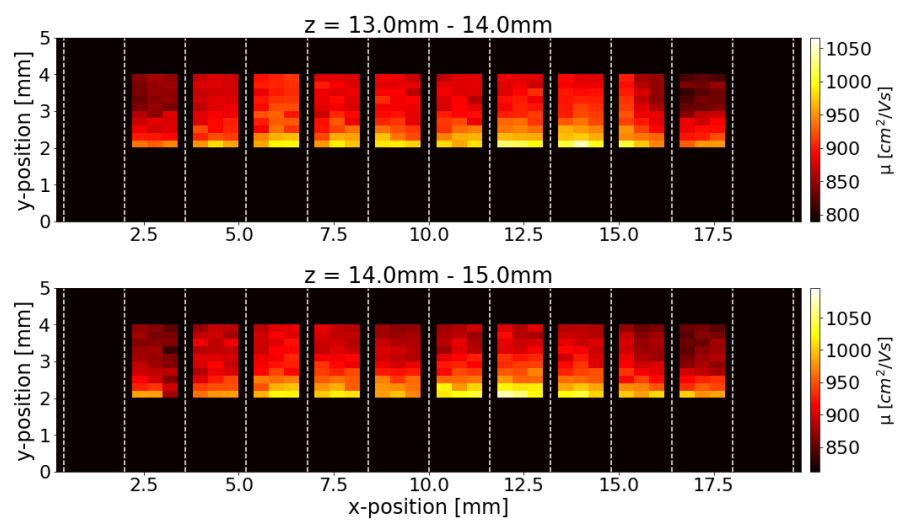

Fig. 10. $\mu_{e}$-map of the chosen detector subvolume. The mean estimated error of the calculated $\mu_{e}$-values is $\sim 10 \%$. White dashed lines: Drift cell boundaries.

\section{Implementation in the $3 D$ CZT detector model}

The next step is to investigate how the newly generated $3 \mathrm{D}$ maps affect the performance of the 3D CZT detector model. In this article, one example of the comparison between a real event and the detector model performance will be presented. Fig. 11 shows the measured pulse shapes for a real event in the 3D CZT detector. To simulate this event, we calculate the $\mathrm{x}-$, $y$-, and z-position alongside the start time of the event. This is then used as input for the 3D CZT detector model. This will of course carry the error of the calculation in position and start time into the modelled data, however, this should only introduce small disagreements between model and data. The model then extracts the $\mu_{e}$ and $\tau_{e}$ values representing the voxel from which the position of the event originates, from the calculated 3D maps, subsequently, the model was run; simulating the event. The event was simulated using two sets of $\mu_{e^{-}}$and $\tau_{e^{-}}$ values. The ones provided by the manufacturer, and the ones from the calculated 3D maps. For the hole material properties, the hole mobility $\left(\mu_{h}\right)$ and lifetime $\left(\tau_{h}\right)$ values used are the ones provided by the manufacturer in Table III, assuming uniform values. Fig. 12 shows a comparison of the real data and the two models, including the main triggered electrodes. Inspecting the three anode signals (Fig. 12(a-c)), we conclude that the model using the newly calculated 3D maps improves the modelled signal greatly, compared to the model using the uniform value provided by the manufacturer. Consequently, the modelled transient signals on the neighbouring anodes are improved. Investigating the cathode signals (Fig. 12(df)), we clearly observe that the model using the 3D maps, simulate the slope of the cathode signal much better than the manufacturer values. We do see however, that the real data have an increase in the cathode signal after electron collection, that does not occur in the model. This indicates that the hole mobility and lifetime is better than what was provided by the manufacturer. This invites for a future characterization of the hole mobility and lifetime in the 3D CZT drift strip detector.
Finally, comparing the drift signals (Fig. 12(g-j)), once again we see that the signals are represented much better by the model using the 3D maps than the one using the uniform manufacturer values.

We clearly see that the model demonstrates a great improvement in prediction when applying the calculated 3D maps compared to using a uniform assumption with manufacturer provided values. Currently the discrete values for a given voxel extracted from the 3D maps, are used as model input for all interaction positions within that voxel. However, in reality the $\mu_{e} \tau_{e}, \tau_{e}$, and $\mu_{e}$ values should vary gradually throughout the material. Interpolation of the $3 \mathrm{D}$ maps could be implemented in the future.
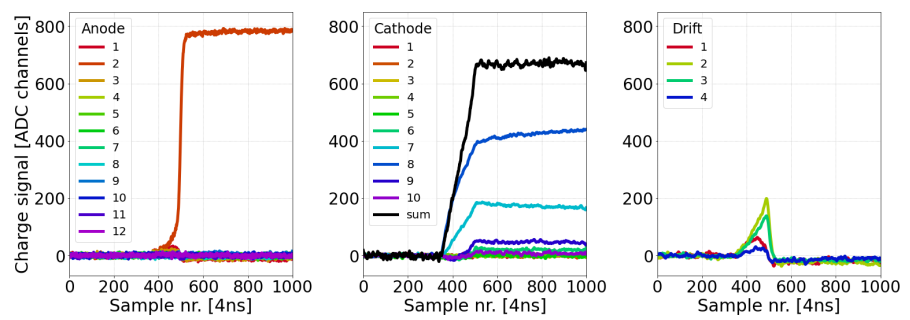

Fig. 11. Anode, cathode, and drift pulse shapes of a real event. $x=2.66 \mathrm{~mm}$, $y=3.95 \mathrm{~mm}, z=14.52 \mathrm{~mm}$, and $t_{0}=352.6$ samples.
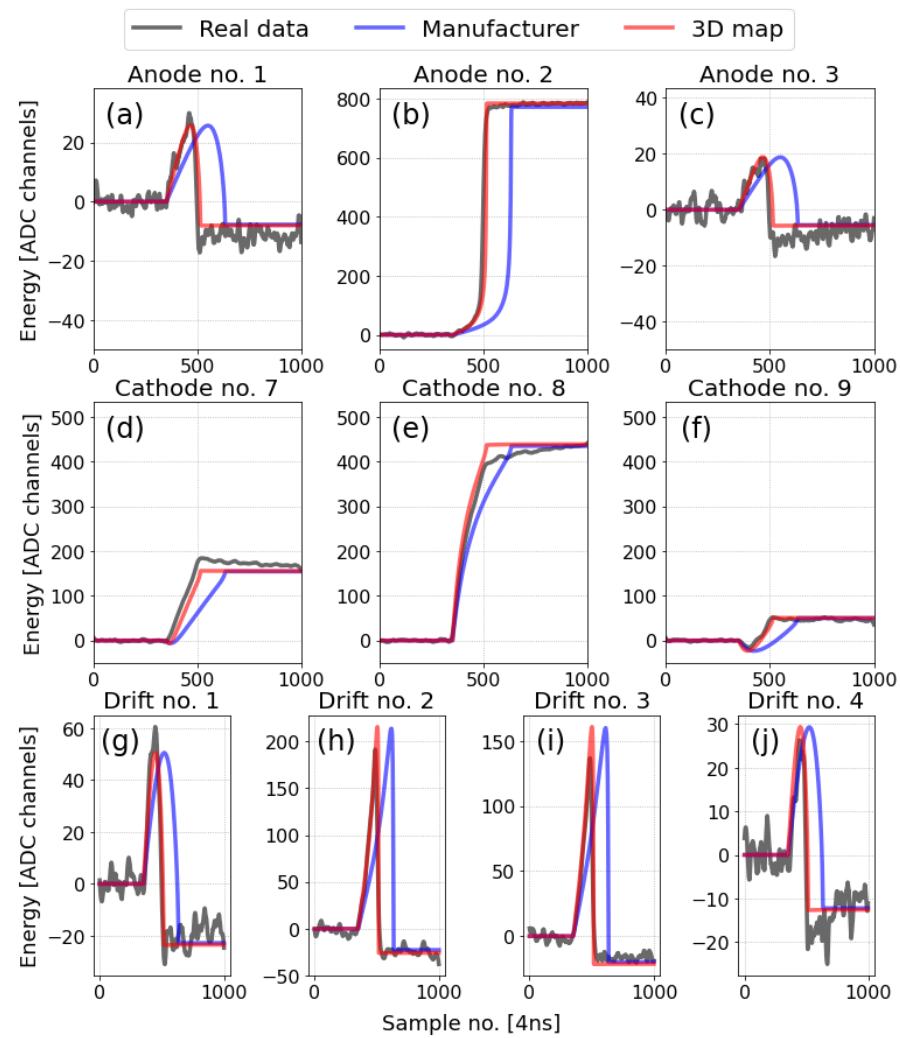

Fig. 12. Comparison between pulse shapes of a real event, and pulse shapes simulated by the model using manufacturer provided values of $\mu_{e}$ and $\tau_{e}$, and the model using the $\mu_{e}$ and $\tau_{e}$ values extracted from the calculated 3D maps. 


\section{CONCLUSION}

Utilizing the 3D position and spectroscopic capabilities of the 3D CZT drift strip detector, a material analysis was performed, to create $3 \mathrm{D}$ maps of the detector material electron mobility and lifetime. This resulted in three 3D maps for a subsection of the detector volume; a map of the $\mu_{e} \tau_{e}$-product, a map of $\tau_{e}$, and finally a map of $\mu_{e}$. The 3D maps were tested, simulating real data events, using the 3D CZT detector model first using the uniform $\mu_{e}$ and $\tau_{e}$ values provided by the manufacturer, and second simulating the event using the newly calculated 3D maps. Comparing modelled pulse shapes with the pulse shapes of the real event, the model demonstrated a great improvement when applying the calculated 3D maps. It also did show that the current assumptions of the holematerial properties (manufacturer provided) did not represent the detector material. We can therefore conclude that the newly calculated 3D maps have greatly improved the 3D CZT detector model performance, to produce more reliable predictions. Not only will these 3D maps provide better possibilities for data correction in the 3D CZT drift strip detector; a model that correctly simulates the pulse shapes makes it possible to generate training data for artificial neural networks.

\section{REFERENCES}

[1] Z. He, F. Knoll and D. K. Wehe, "Direct measurement of product of the electron mobility and mean free drift time of CdZnTe semiconductors using position sensitive single plarity charge sensing detectors," Journal of Applied Physics 84, 5566, 1998.

[2] A. E. Bolotnikov, G. S. Camarda, E. Chen, R. Gul, V. Dedic, G. De Geronimo, , J. Fried, A. Hossain, J. M. MacKenzie, L. Ocampo, P. Sellin, S. Taherion, E. Vernon, G. Yang, U. El-Hanany, and R. B. James, "Use of the drift-time method to measure the electron lifetime in longdrift-length CdZnTe detectors," Journal of Applied Physics 120, 104507, 2016.

[3] L. Abbene, G. Gerardi, F. Principato, A. Buttacavoli, S. Altieri, N. Protti, E. Tomarchio, S. Del Sordo, N. Auricchio, M. Bettelli, N. Sarzi Amadé, S. Zanettini, A. Zappettinig, and E. Carolif, "Recent advances in the development of high-resolution 3D cadmium-zinc-telluride drift strip detectors," Journal of Synchrotron Radiation, 27, pp. 1564-1576, 2020.

[4] S. Abbaszadeh, Y. Gu, P. D. Reynolds, and C. S. Levin, "Characterization of a sub-assembly of 3D position sensitive cadmium zinc telluride detectors and electronics from a sub-millimeter resolution PET system," Phys. Med. Biol. 61, 6733-6753, 2016

[5] S. Howalt Owe, I. Kuvvetli and C. Budtz-Jørgensen, "Evaluation of a Compton camera concept using the 3D CdZnTe drift strip detectors," Journal of Instrumentation, 14(01) C01020, 2019.

[6] M. A. J. Van Pamelen and C. Budtz-Jørgensen, "Novel electrode geometry to improve performance of CdZnTe detectors," Nuclear Instruments and Methods in Physics Researchs Section A: Accelerators, Spectrometers, Detectors and Associated Equipment. Elsevier 403, 2-3, pp. 390-398, 1998.

[7] M. A. J. Van Pamelen and C. Budtz-Jørgensen, "CdZnTe drift detector with correction for hole trapping," Nuclear Instruments and Methods in Physics Research Section A: Accelerators, Spectrometers, Detectors and Associated Equipment 411.1 pp. 197-200, 1998.

[8] I. Kuvvetli et al., "A 3D CZT high resolution detector for $\mathrm{x}$ - and gamma-ray astronomy," High Energy, Optical, and Infrared Detectors for Astronomy VI. SPIE Astronomical Telescopes + Instrumentation, Montréal, Quebec, Canada. 2014, pp. 91540X.

[9] C. Budtz-Jørgensen and I. Kuvvetli, "New Position Algorithms for the 3-D CZT Drift Detector," IEEE Transactions on Nuclear Science, 64.6, pp. 1611-1618, April 2017. 